\title{
Panorama de la robótica colombiana en ámbitos sociales
}

\author{
Yeyson Alejandro Becerra Mora*
}

\section{Resumen}

Colombia ha entrado en una era de posconflicto en la que aún permanecen desafíos que deben ser resueltos para mejorar el bienestar de la sociedad. En este artículo se presentan tres casos distintos de impacto social en Colombia, a saber, desactivación de minas antipersona, desarrollo de prótesis para personas afectadas por el conflicto armado y agroindustria en el posconflicto; y los aportes brindados por las instituciones de educación superior, el sector privado y el sector público, a través del uso de la robótica como parte de la solución a estas problemáticas.

\section{Abstract}

Colombia has entered a post-conflict age, in wich still remain some challenges that should be resolved in order to improve society wellness. This article presents three different social impact cases in Colombia, deactivation of antipersonnel mines, prosthesis development for affected people by the armed conflict, and agroindustry in the Colombian post-conflict, and the contributions provided by higher education institutions, private and public sector, adopting robotics as part of the solution to these problematic.
Cómo citar este artículo (APA): Becerra, Y. (2019). Panorama de la robótica colombiana en ámbitos sociales. Hashtag, 14, 85-101.

> Palabras clave: agroindustria colombiana, minas antipersona, robótica, posconflicto colombiano, prótesis

> Keywords: Antipersonal Mines, Colombian Agroindustry, Colombian Post-Conflict, Robotics, Prosthesis

\footnotetext{
Corporación Unificada Nacional de Educación Superior, cun. Contacto: yeyson_becerra@cun.edu.co
} 


\section{Introducción}

$\mathrm{L}$ a robótica es una de las tecnologías presentes en la cuarta revolución industrial, que está cambiando el mundo. Muchas personas piensan que, con la llegada e implementación de nuevas tecnologías, muchos trabajos desaparecerán, lo que dejaría a millones de personas desempleadas alrededor del mundo. Pero lo cierto es que la sociedad tiene la oportunidad de avanzar, como ha pasado en otras épocas, y crear nuevos trabajos en los distintos sectores de la economía. También es cierto que dichos trabajos vendrán con nuevos desafíos para la sociedad, ya que la población tendrá que adaptarse a los cambios propuestos por la tecnología y continuar desarrollando sus capacidades cognitivas. Esto se puede sostener en que, por ejemplo, las tareas repetitivas y riesgosas para el ser humano han venido siendo tomadas por los robots en los últimos años y las proyecciones indican que lo seguirán haciendo.

En ese sentido, la cuarta revolución industrial es una realidad que Colombia tiene que adaptar para ser más competitivo en el mundo. Si bien ya se ha comenzado a hablar de las ventajas que trae adoptar tecnologías como la robótica, aún falta bastante recorrido para apropiarse de estos conocimientos, tal y como como ya lo han hecho países desarrollados.Dado que Colombia sigue siendo un país en vías de desarrollo, aún necesita invertir más en actividades de investigación y desarrollo. Según datos del Banco Mundial, la inversión realizada por el país en este ámbito fue de apenas $0,24 \%$, por debajo de países como Argentina y Brasil, y lejos del promedio de los países que pertenecen a la Organización para la Cooperación y Desarrollo Económico (OCDE).

Actualmente, en Colombia un mayor número de programas profesionales, tecnólogos y técnicos han orientado sus planes de estudio a las áreas de la robótica, al reconocer el futuro prometedor e impacto que está teniendo y tendrá esta tecnología alrededor del mundo. Las instituciones de educación superior (IES) no solo han venido creando cursos orientados a la robótica, sino que además han estado promoviendo la investigación en ello, con semilleros y grupos de investigación.

En esa misma línea, la industria colombiana ha empezado a prestar atención a la robótica en los últimos años, especialmente a la robótica industrial, con el fin de mejorar sus niveles de producción y ser más competitivos tanto al interior del país como en el exterior. Sin embargo, toda esta tecnología ha sido importada de países como Alemania y Japón, que presentan los mayores índices de ventas en robots industriales, lo que deja a Colombia como un país con empresas que solo se limitan a comercializar y dar soporte en ello.

Por el lado de la robótica de servicio, el panorama es un poco más alentador, gracias a que algunas IES se han comprometido con proyectos de investigación que le aportan al desarrollo colombiano, al ofrecer soluciones a problemáticas de la sociedad. Cabe destacar que algunos robots orientados al hogar, entretenimiento, inspección aérea, inspección terrestre, educación, entre otros, han venido siendo importados de países europeos y de Estados Unidos, siendo este último uno de los grandes representantes de la robótica de servicio alrededor del mundo.

En este artículo se mostrarán tres casos en los que la robótica de servicio ha sido de ayuda para el desarrollo del país, y cómo las IES, el sector privado y el gobierno han aportado en cada una de estas iniciativas. Los tres casos seleccionados por su impacto social fueron: identificación, desactivación y/o destrucción de minas antipersona en territorio colombiano, adelantos de prótesis en 
Colombia para personas afectadas en el conflicto armado y el renacer de la agroindustria en la era del posconflicto. Cada uno de estos temas fue abordado desde una perspectiva tecnológica, cuyo eje principal fue a la robótica.

\section{Identificación, desactivación y/o destrucción de minas antipersona en territorio colombiano}

Colombia ha estado entre los mayores sembradores de minas antipersona en el mundo. Según datos recopilados por la Unicef en el 2000, se estimó que el país tenía plantado alrededor de 100000 minas antipersona, a lo largo del territorio nacional, contando entre las fabricadas dentro y fuera del país, así como las fabricadas artesanalmente. En ese mismo informe, la Unicef agregó el alarmante dato de crecimiento de la plantación de minas en un 20 \% anual (Unicef, 2000).

Para el 2010, Colombia ocupó el segundo lugar de países en el mundo en donde que más personas se vieron afectadas debido a estos artefactos
(570 casos), superado únicamente por Afganistán. En el 2017, el país descendió al décimo puesto, al tener una disminución de un $90 \%$ en el número de victimas reportadas (56 casos), con respecto al 2010. Según registros del Comité Internacional de la Cruz Roja, Colombia prácticamente había duplicado el número de víctimas en los meses de enero a agosto del 2018 (99 casos). Sin embargo, desde las negociaciones entre el Gobierno Nacional de Colombia y el grupo subversivo de las Fuerzas Armadas Revolucionarias de Colombia (FARC), que dio como resultado la firma del acuerdo de paz entre ambas partes, el número de víctimas por minas antipersonales ha venido decreciendo en el territorio nacional.

Figura 1. Víctimas de minas antipersona

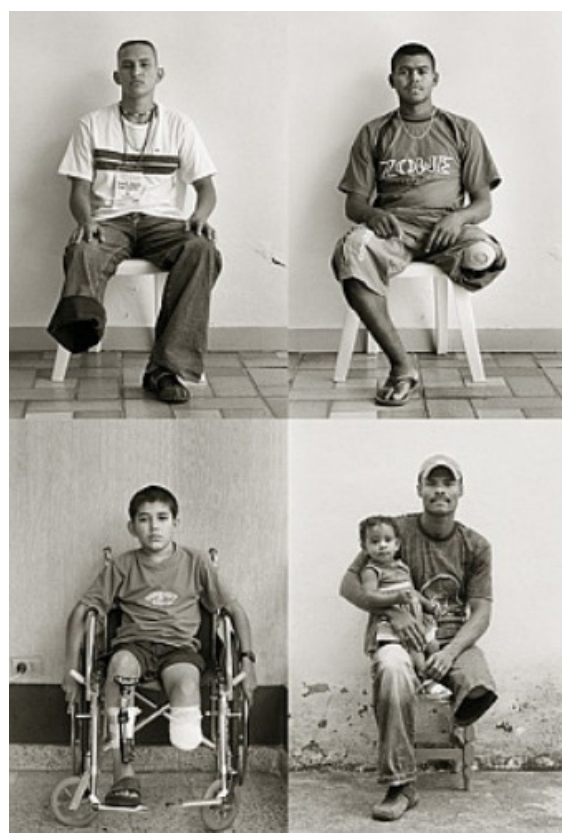

Fuente: Gaviria (2014) 
Las minas antipersona han dejado miles de víctimas a lo largo de los años en Colombia. Un informe de la organización Descontamina Colombia comunicó que entre 1990 y 2017 se registraron 11585 víctimas a causa de estos artefactos (Organización Descontamina Colombia, 2018). Algunas técnicas que se han venido empleando regularmente en el desminado han sido medios manuales, mecánicos y caninos. La persona que realiza la labor de desminado a través de medios manuales corre un alto riesgo de ser afectado por el artefacto. Aunque utilizar medios mecánicos reduce este riesgo, la persona debe estar próxima a la mina para poder desactivarla, corriendo el riesgo de tropezar con otra en los alrededores. Por último, queda el medio canino, siendo, de estos tres, el más seguro para las personas.

El Centro Nacional contra Artefactos Explosivos y Minas del Ejercito Nacional (Cenam) reportó que entre 2003 y 2015 fueron desactivados 224254 artefactos explosivos (minas antipersona, municiones sin explosiones y artefactos improvisados). Uno de los compromisos de las FARC en el acuerdo de paz fue apoyar labores humanitarias para la identificación de minas antipersona en el país, con lo cual se espera que el número de estos artefactos sembrados a lo largo del territorio colombiano disminuya considerablemente desde 2018 en adelante.

En la década de los 70, los grupos subversivos comenzaron a emplear minas antipersona en territorio colombiano, por lo que Colombia las ha padecido por más de cuatro décadas. Solo hasta principios de la década pasada, Colombia comenzó a realizar investigación en métodos no convencionales, como fueron la construcción de robots móviles para la desactivación de minas antipersona, pero fue desde 2012 que el Gobierno Nacional impulsó la investigación en métodos distintos a los manuales, mecánicos y caninos.

Con el fin de evitar un mayor número de víctimas cuando se está en el proceso de búsqueda y desactivación de las minas antipersona, se empezaron a diseñar y desarrollar vehículos aéreos no tripulados, $\mathrm{UAV}^{1}$; robots móviles teleoperados, robots móviles con manipuladores, entre otros, para el apoyo de labores de desminado.

Como se había mencionado previamente, uno de los principales intereses en utilizar robótica en los procesos de desminado fue salvaguardar la vida de las personas que se encuentran en estas labores. Con este principio, la universidad EAFIT en el 2015 elaboró dos robots móviles con un mecanismo para activar las minas, hacerlas explotar de manera controlada y minimizar el riesgo para los soldados. Este desarrollo fue probado en la vereda El Orejón, municipio de Briceño, Antioquia, para la reactivación de labores de desminado después del fallecimiento de un soldado a causa de la explosión de unos de estos artefactos (El Colombiano, 2015).

La investigación realizada en la Universidad EAFIT para apoyar las labores de desminado en territorio colombiano arrojó un spin-off que se denominó Tecnologías MARTE S.A.S., dedicado a la creación de tecnología orientada al sector militar. Entre sus productos se encuentran robots móviles para el desminado mecánico, manipulación de explosivos e inspección.

El objetivo de una mina antipersonal no es matar a la víctima, si no que esta quede herida en el campo de batalla para que posteriormente otra persona se acerque a socorrerla, lo que reduce el número de combatientes. En la actualidad, son pocas las minas antipersona que utilizan metal en su elaboración. En vez de ello, son

1 UAV, por sus siglas en inglés: Unnamed aerial vehicle 
construidas con botellas plásticas, tubos de PVC, envases de vidrio y galones plásticos. Además, utilizan material orgánico que es fácilmente adquirible, como gasolina y fertilizante de productos agrícolas.

Figura 2. Minas Antipersonal

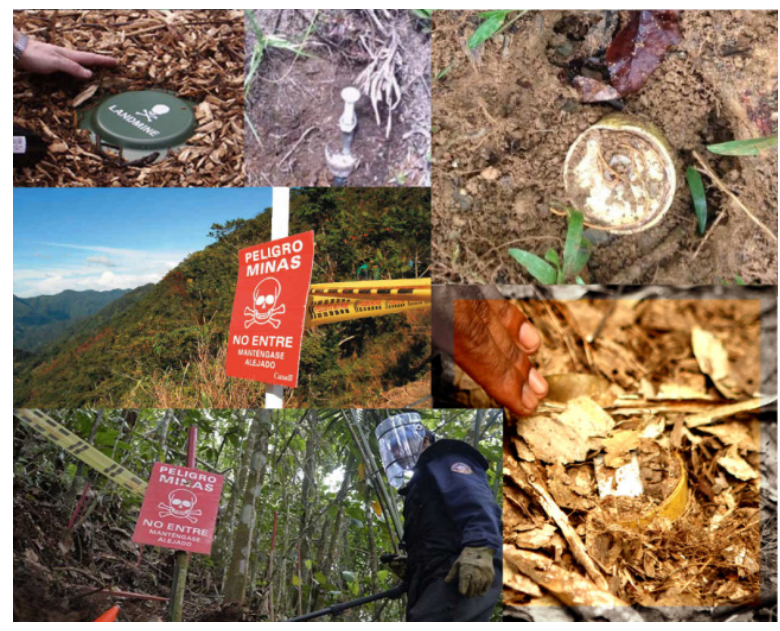

Fuente: elaboración propia.

Teniendo en cuenta los tipos de minas antipersona, se han empleado robots móviles (aéreos y terrestres) equipados con distintos tipos de sensores que han permitido detectar materiales sospechosos bajo tierra. Regularmente se han utilizado detectores de metales para este fin, $y$, de hecho, las minas antipersona hechas con metal son las más perjudiciales una vez activadas, ya que no solo afectan a la quien la pisó, sino a las personas próximas a ella. Sin embargo, las minas antipersona han evolucionado, lo que desafía la detección.

El grupo de física nuclear de la Universidad Nacional de Colombia pensó en un sensor que permitiera identificar irregularidades o material no convencional bajo tierra. Este desarrollo fue hecho en el 2014 y las pruebas demostraron que se podían detectar minas antipersona de 100 gramos y hasta 12 centímetros de profundidad. La tecnología se basa en la utilización de neutrones y rayos gama. En el 2018, ese mismo grupo implementó su sensor en un robot móvil teleoperado que, a medida que avanza en la recolección de datos, refina sus capacidades para encontrar explosivos bajo tierra (MinCiencias, 2018). Actualmente, el robot realiza pruebas con la Industria Militar Colombiana (Indumil).

Otro adelanto por parte de la Universidad Nacional en el 2018 fue el de un robot móvil teleoperado con sistema de tracción tipo trípode. Se usó este diseño con el fin de soportar con mayor eficacia el impacto de la onda (Universidad Nacional de Colombia, 2018). El robot fue construido con material Kevlar, (el mismo utilizado en chalecos antibalas) en su carcasa y caucho de llantas reutilizadas. Además, utiliza radiofrecuencia para ser controlado. El equipo afirma que los equipos provenientes del extranjero son poco útiles debido al desconocimiento de que estos tienen acerca de la topografía colombiana.

Para el 2011, la Universidad Javeriana presentó a Arcadio, un robot teleoperado de 70 kilogramos, que permite identificar cambios en el suelo 
y extraer elementos peligrosos con su manipulador. El grupo de investigación que lo desarrollo manifestó la relevancia de utilizar un sistema como este en favor de preservar la vida e integridad del ser humano (El Mundo, 2011).

La cooperación entre el sector académico (Universidad Militar Nueva Granada) y de defensa (Indumil) dio como resultado a VALI (Vehículo Antiexplosivo Ligero). VALI es un robot móvil teleoperado con un alcance inalámbrico de hasta 76 metros y 70 kilogramos de peso, dotado con un brazo manipulador, tres cámaras y un cañón irruptor que dispara una carga no letal (polímero, plastilina o agua), (Medellin, 2015). Su costo ronda los 200 millones de pesos.
En los últimos años, el uso de UAV, más conocidos recientemente como drones, se ha proliferado considerablemente debido a las distintas aplicaciones que se les ha encontrado, entre ellas, la detección e inclusive destrucción de estos artefactos. La Universidad Javeriana de Cali, en el 2014, planteó el proyecto DUBO para el desminado colombiano (Tobon, 2014). Este constaba de tres partes: comprender el entorno electromagnético en el cual están ubicadas las minas, utilizar radar de bajo costo que pueda ser acoplado en un UAV y desarrollar un algoritmo que permita realizar la detección de la mina en el mismo sitio.

Figura 3. Detección y destrucción de minas antipersonal utilizando uAV

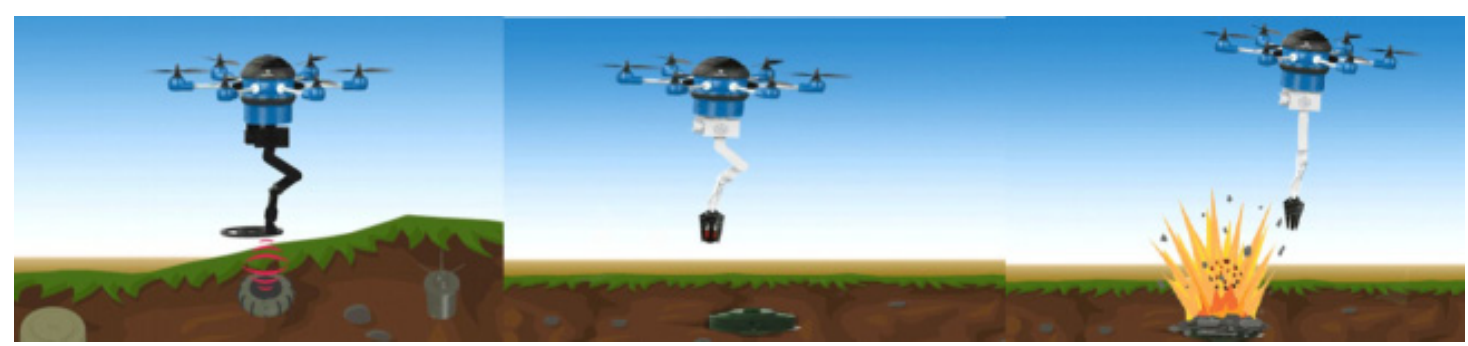

Fuente: Kickstarter (s.f.)

Las investigaciones realizadas en la Universidad Javeriana dieron más claridad al objetivo del proyecto, al orientarlo al " desminado en territorio colombiano". El UAV utilizado en el grupo de investigación del departamento de electrónica para tareas de desminado (Mayorga, 2015) cuenta con GPR (Ground Penetrating Radar) y visores para reconocimiento del entorno, además de un mando a distancia para que este sea teleoperado. La cámara permite georreferenciar el terreno, así como identificar patrones de ubicación, mientras que el radar emite una señal que penetra la tierra e indica al rebotar si existe una mina. El sistema además cuenta con un algoritmo que utiliza técnicas de visión por computador para generar una imagen de mosaico del terreno cubierto, lo que detecta en tiempo real objetos parecidos a minas terrestres (Colorado, Mondragon, Rodriguez y Castiblanco, 2015). Las pruebas realizadas hasta el 2015 fueron desarrolladas en terrenos estructurados. El grupo de investigación propuso, para futuras investigaciones, la miniaturización de componentes electrónicos, evasión de obstáculos e identificación de objetos distintos a minas en tiempo real.

Otro sistema compuesto por UAv fue el premiado en el reality El Gran Inventor Colombia (El Colombiano, 2016). El sistema localiza y detona la mina de una manera controlada sin necesidad 
de alambres. La localización de la mina se realiza a través de una nariz electrónica, que reconoce los elementos con los que son elaboradas las minas antipersona. La detonación de la mina se realiza por medio de pequeños explosivos que se dejan caer sobre la mina una vez esta sea debidamente localizada. La estructura mecánica del prototipo fue elaborada en aluminio y fibra de carbono; el control se realiza a través de un mando a distancia.

Desde que inició el proceso de paz, el Gobierno de Colombia ha destinado recursos a investigación para que las universidades se comprometan con el desarrollo de tecnología que ayude al desminado en el país. Sin embargo, la recopilación de información expuesta en este capítulo muestra que la gran mayoría de desarrollos fueron hechos en Bogotá, por instituciones como la Universidad Nacional, la Universidad Javeriana, la Universidad Militar Nueva Granada y el SENA.

También se han realizado avances significativos en la identificación de minas, gracias a sensores como el GPR o el sensor que utiliza neutrones y rayos gama para detectar material sospechoso bajo tierra. El sensor de metal utilizado con anterioridad quedó obsoleto una vez que la mina antipersona evolucionó. Además, la robótica no solo provee sistemas terrestres como los

\section{El renacer de la agroindustria en la era del posconflicto}

La ingeniería tiene un rol importante en el desarrollo del país y en el bienestar de la sociedad. Afectada por el conflicto armado, la agroindustria colombiana ha tenido un rezago en el aumento de niveles de producción de alimentos, con millones de hectáreas improductivas o utilizadas para cultivos ilícitos. A pesar del potencial vehículos terrestres no tripulados, $\mathrm{UGV}^{2}$, sino también los UAV.

La robótica en este campo seguirá siendo una tecnología que continuará salvando vidas y que contribuye al propósito de tener una Colombia libre de minas antipersonal y tal como lo propuso el expresidente Juan Manuel Santos durante su Gobierno. Se espera que, para el 2021, Colombia sea un país libre de estos artefactos. No se puede continuar pensando que estas labores sean exclusivas del ser humano, por lo cual Colombia se debe apropiar del uso de la tecnología para avanzar en las labores de desminado que protejan la vida humana.

La optimización de las labores del robot para el desminado aún tiene muchos desafíos como, por ejemplo, hacerlos más autónomos y evitar que sean teleoperados, como se vio en la gran mayoría de estos. También se debe mejorar su aprendizaje del entorno, esto con el fin de aumentar el número de aciertos en la detección de minas; diseñar y construir sistemas mecánicos más robustos que permitan al robot desempeñarse en la geografía colombiana; y, por último, reconocer y eludir agentes dinámicos, por ejemplo, animales y/o personas, que puedan aparecer durante las labores de desminado.

que Colombia tiene en la agroindustria, este no ha sido debidamente aprovechado, como otros países alrededor del mundo si han aprovechado el suyo. Según lo comentan Méndez-Fajardo y Pérez (2015), una vez finalizara el conflicto con las FARC, se preveía una reactivación de este sector, teniendo en cuenta la restitución de tierras 
e inversiones en tecnología que le permitirían al país aumentar la autosuficiencia en alimentos, así como las oportunidades de entrar a mercados internacionales.

En el 2016 se estimó que, de los suelos netamente agrícolas, que son 11.3 millones de hectáreas, no se aprovechaban el 65,8 \% (El Tiempo, 2016a); además, se importaba aproximadamente el 28,5\% de alimentos consumidos en hogares. Con el final del conflicto armado en este mismo año, se previó el gran potencial de Colombia en la agricultura, siendo estas una de las industrias con mayor futuro y que más le aportará al PIB del país, más aún cuando las proyecciones de producción y reservas de petróleo se encuentran a la baja.

Desde la unidad de planificación rural agropecuaria, propusieron crear mapas detallados para identificar qué suelos y climas son los más indicados para cultivos, lo que mostró que productos como aguacate, piña y madera crecerán sustancialmente en exportaciones para los próximos años, por lo cual, el aprovechamiento de tierra debe ser óptimo. El peso de los productos agrícolas en las exportaciones realizadas en el 2017 fue de tan solo 8 \%, siendo el café y el banano los productos más representativos. Los productos agrícolas de países como Chile y Argentina pesaron el $14 \%$ y 50 \% respectivamente, del total de las exportaciones realizadas en ese mismo año (Bloomberg, 2017).

Dos problemas que se evidenciaron para que Colombia tuviera bajos niveles de exportaciones agrícolas a pesar de su gran potencial fueron bajos niveles de tecnología, debido a la presencia de grupos subversivos en los sectores rurales del país, y altos costos de transporte. Sin embargo, estos dos problemas han venido siendo subsanados. Para el primero, se firmó un acuerdo de paz que desmovilizo a miles de guerrilleros; para el segundo, se ha venido avanzando en la construcción de vías para el desarrollo del país en los últimos años.

La población para el 2050 se situará en torno a los 9000 millones de personas, lo que significa que se consumirá un 50 \% más de alimentos de los consumidos actualmente (Colcapital, 2015), por lo cual Colombia podría llegar a ser una potencia en el sector agroindustrial, si se hacen los debidos ajustes en este momento. Además de realizar la restitución de tierras, fortalecer la infraestructura del país y formalizar la tenencia de tierra y los canales de comercialización, se debe pensar en realizar una importante inversión en tecnología que lleve a Colombia a ser un país más competitivo respecto a los países con los que tiene Acuerdos de Libre Comercio vigentes.

En el sector agroindustrial se debe pensar en realizar labores de una forma más rápida, económica y periódica, para lo que un sistema como el UAV puede ser de ayuda. Tener acceso a este tipo de tecnología permitirá que nuevas generaciones permanezcan en el campo, dado que esta traerá mejores ingresos, lo que se traduce en un mayor impacto social para el país. La apropiación de esta tecnología hará que residentes de las zonas rurales sean los operarios de estos sistemas (UAV), lo que creará nuevos puestos de trabajo acordes a la cuarta revolución industrial y, así mismo, generará inversión social gracias a estas nuevas tecnologías.

Colombia, al ser país un tropical, tiene la ventaja de estar cultivando y cosechando durante todo el año, a diferencia de los países con estaciones. La desventaja para Colombia es no tener la tecnología apropiada para aumentar su productividad para estar entre los mayores productores de alimentos en el mundo.

Además de utilizar la tecnología para aumentar la productividad, también es posible utilizarla para realizar tareas nocivas para la salud 
humana, como la aplicación de herbicidas, lo que hace más eficiente la fumigación, a diferencia de la forma en que las avionetas la hacen.

En los últimos años se ha empezado a masificar el concepto de la agricultura de precisión, del cual los UAV hacen parte. Los UAV en el sector agroindustrial pueden ser controlados por un operador o pueden ser autónomos. Están provistos de cámaras visuales, infrarrojas y multiespectrales o térmicas. Las cámaras visuales permiten tomar fotos georreferenciadas para identificar área efectiva, caminos, agua y realizar la logística de un amplio terreno. La información puede ser almacenada para ser consultada posteriormente $y$, mediante algoritmos de visión por computador, se puede calcular la densidad de siembra, así como la cantidad de árboles y plantas de forma automática. Las cámaras multiespectrales obtienen los colores que refleja la planta a través de una firma espectral, que está compuesta por características únicas e inequívocas de la planta. Con esto se identifica si la planta está saludable, con estrés hídrico o si tiene alguna maleza. Además, con las cámaras multiespectrales se puede generar mapas de diagnóstico, como también de condiciones de cultivo, de fertilizantes, de contenido de nitrógeno, de estrés hídrico y de plagas.

Figura 4. uav en la agroindustria

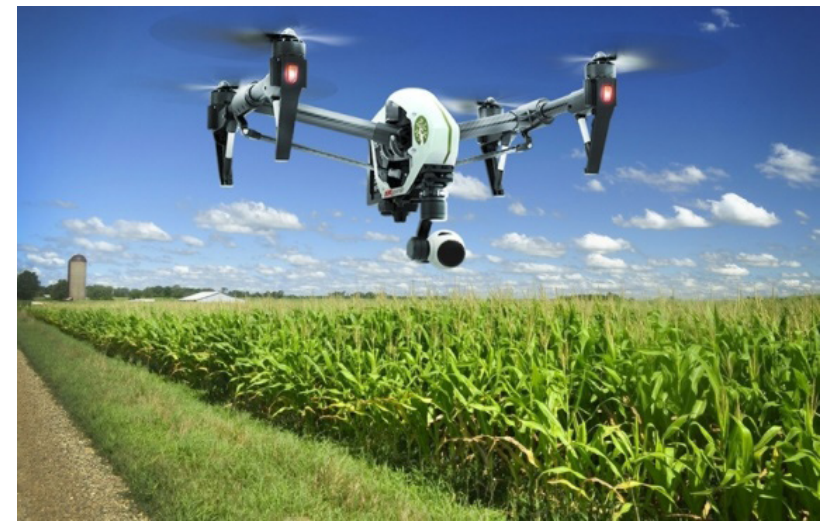

Fuente: Infodron (2016)

Con el desarrollo de esta tecnología, se esperan ahorros financieros y menor impacto ambiental. Para el 2015, se encontró que el 80 \% del capital invertido en UAV estaba enfocado en la agricultura (ASOHOFRUCOL, 2015), siendo Estados Unidos y Europa los mayores productores de estos artefactos. Los costos para ese año rondaban los 20000 y 30000 dólares, a los que hay que sumarle los costos de mantenimiento. La utilización de los UAV se encuentra orientada a terrenos de muchas hectáreas, como son los cultivos de cereales, trigo y legumbres.
Uno de los cultivos más representativos en Colombia es la caña de azúcar, para los que se ha estado evaluando el uso de cámaras multiespectrales en UAV que usen la técnica de ortofotos (mosaicos georreferenciados), para así obtener información topográfica en la delimitación de áreas, actualización cartográfica, cuantificación de áreas infestadas por malezas, cuantificación de áreas de resiembra y digitalización de líneas de surcado. Con dicha técnica también se obtienen modelos digitales de elevación para estimar curvas a nivel, construir mapas de pendientes, modelos de superficie y modelos para estimar la 
altura del cultivo de la caña de azúcar, así como registrar energía infrarroja para el seguimiento de variables biofísicas. Se han encontrado correlaciones del $74 \%$ con un error de $\pm 25 \mathrm{~cm}$ entre la altura tomada en campo y la calculada por el modelo (Garcia, s.f.). En los cultivos de caña de azúcar se busca identificar y cuantificar zonas con problemas de bajo desarrollo fisiológico y corrección de variables como concentración de clorofila y contenido de nitrógeno.

Figura 5. Cámara multiespectral

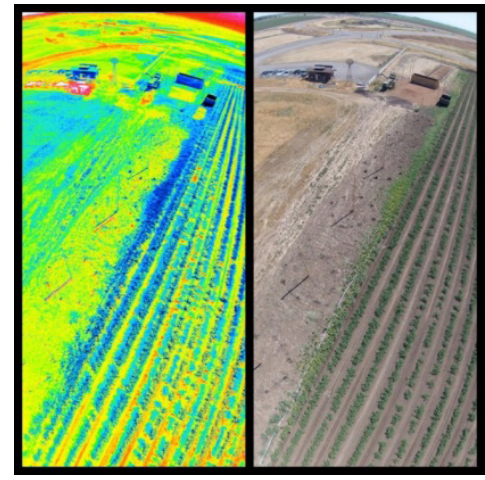

Fuente: Gonzoearth (2013)

La investigación realizada por el International Water Management Institute (IWMI) y dirigida por Salman Siddiqui (The Guardian, 2015), director del departamento de detección remota, mostró la relevancia de utilizar UAV equipados con sensores infrarrojos para detectar el estrés de una planta (falta de agua, fertilizantes o el ataque de una peste), hasta 10 días antes de que el ojo humano lo pueda hacer. Siddiqui concluye en su investigación que "la detección temprana de estrés previene perdidas a gran escala" (The Guardian, 2015).

Entre los UGV construidos en Colombia, como fruto de la cooperación entre la Universidad Nacional de Colombia y la Universidad Militar Nueva Granada, encontramos la plataforma robótica Agrobot CERES (González, 2018), la cual fue diseñada y construida para destruir malezas, agregar nutrientes y aplicar fungicidas. La Universidad Militar Nueva Granada se encargó del diseño mecánico y la adecuación de motores, mientras que la Universidad Nacional de Colombia se encargó del sistema de movimiento con motores lineales, dos de ellos se mueven en un plano $x$ ey sobre el cultivo, mientras el tercero se emplea sobre un eje $z$. El robot fue pensado para que se desplazara sobre los surcos del cultivo. Robots como Agrobot CERES, utilizan láser, cámaras y sensores infrarrojos para detectar la maleza, y un brazo mecánico para la aplicación de herbicidas, fungicidas y remoción de maleza.

Figura 6. Agrobot CERES

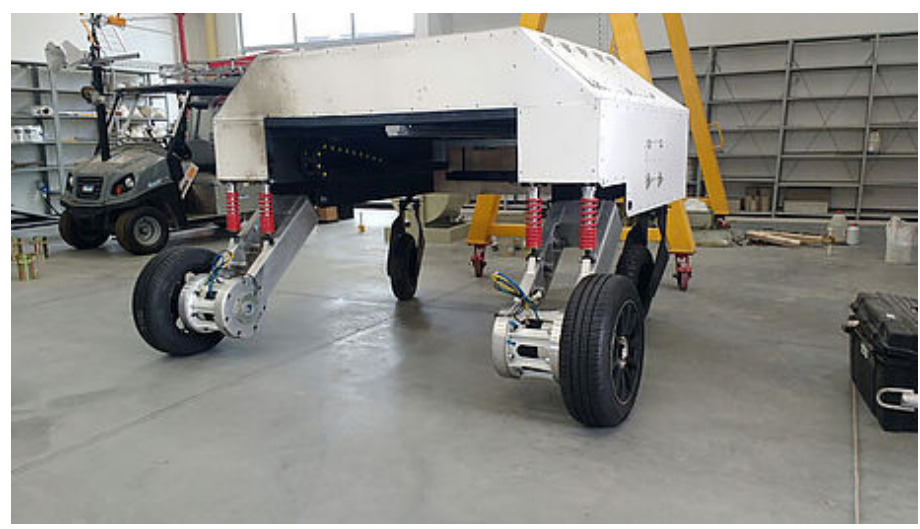

Fuente: Gonzáles, 2018. 
Algunas ventajas que se proyectan con el uso de robots en la agroindustria son las de producir alimentos en cantidad y calidad de una forma sostenible y respetuosa; tener un sector agrícola más competitivo, con mayor demanda y mayor calidad de alimentos; mejorar la respuesta en los cultivos y en calidad ambiental; incrementar la rentabilidad, calidad, cantidad y rendimiento en los cultivos; contar con una tecnología en permanente desarrollo; tener la oportunidad de monitorear, registrar y evaluar variables; tener cultivos eficiente y sustentables; y ajustar las prácticas de cultivo según las necesidades de la planta.

Países como Estados Unidos, Canadá y Australia han sido pioneros alrededor del mundo en la adopción de tecnologías para el sector agroindustrial, mientras que, en Latinoamérica, lo han sido Argentina y Brasil. En la agricultura tradicional los campos son homogéneos, no se incluye variabilidad espacial, ni temporal, ni el análisis para la aplicación de insumos. Pero en la agricultura de precisión se habla de una optimización de recursos, tanto ambientales como energéticos; se tiene en cuenta la variabilidad espacial y las dosis acordes para la siembra; se reducen costos en insumos, pesticidas y fertilizantes; se aumenta el rendimiento con el mismo nivel de insumos y la calidad de las cosechas; se obtienen productos de mayor valor nutritivo, se controlan los cultivos en tiempo real y se evitan problemas de salud ocupacional. Todas estas ventajas mencionadas deben ir acompañadas de un mayor trabajo social con agricultores, con el fin de que ellos se apropien y entiendan la tecnología para su uso y beneficio.

Algunas dificultades para producir alimentos de una manera sostenible en países industrializados son: envejecimiento de la población rural; emigración del campo a la ciudad y el escaso margen comercial que le queda al agricultor al final de la cosecha. Se espera que esta tendencia cambie gracias al uso de la tecnología, ya que con esta las proyecciones muestran un mejoramiento en las condiciones de los agricultores y un incentivo para que se mantengan o vuelvan a los campos. Por otro lado, informes del Instituto Global McKinsey y del banco mundial (El Espectador, 2018) sugieren que los sectores agrícolas e industriales tienen un mayor potencial para la automatización por ser tareas repetitivas, lo que genera una preocupación por la mano de obra no calificada, pero a la vez se sugiere capacitación de trabajadores para futuros empleos que aún no existen.

En Colombia se ha realizado poca investigación en el diseño y construcción de UAV, ya que regularmente los grupos de investigación acuden a realizar compras en el extranjero para centrarse en algoritmos de control y algoritmos de visión por computador. Actualmente existe una oportunidad en el desarrollo mecánico de estos robots, como lo han aprovechado algunas startups colombianas que diseñan y construyen sus propios UAV. El desarrollo de esta tecnología permite ofrecer servicios más económicos al sector agroindustrial, a diferencia de traer los robots del extranjero.

Por otro lado, el trabajo hecho en robótica cooperativa en los grupos de investigación colombianos ha sido prácticamente nulo. Las UAV y UGV se mantienen interconectados a través de una red de trabajo que les permite realizar distintas labores en campos de cultivo, tales como, monitoreo, análisis, riego, fumigación, siembra, cosecha, entre otras; todas estas se realizan en paralelo. Las empresas colombianas o startups no han explotado aun esta oportunidad, posiblemente por una falta de inversión, desconocimiento o una combinación de ambas. Sin embargo, se aclara que Colombia lleva en promedio 3 años haciendo uso de robots en el sector agroindustrial, así que aún falta mucho camino por recorrer antes de que esta tecnología se masifique. 


\section{Adelantos de prótesis en Colombia para población vulnerable}

Debido a los altos costos que representa importa prótesis de otros países, ingenieros y científicos colombianos han hecho un esfuerzo para que estas sean más asequibles para sectores sociales de bajos recursos. En el 2016, un estudiante de doctorado de la Universidad Nacional de Colombia comenzó a desarrollar, fabricar y ensamblar prótesis para miembros superiores (CNN español, 2017). El material que utiliza para la elaboración de prótesis es ninjaflex y PET. Estos se introducen en una impresora 3D modificada (resolución de impresión, densidades y piezas mucho más definidas y precisas) para la fabricación de las piezas. La población a la que especialmente se dirige este emprendedor es a la de los niños con escasos recursos, razón por la cual sus diseños son alusivos a superhéroes. Las amputaciones que trata pueden ser congénitas o traumáticas. Actualmente se encuentra constituida la empresa Fabrilab, gracias a esta iniciativa.

Figura 7. Prótesis para miembros superiores- Fabrilab

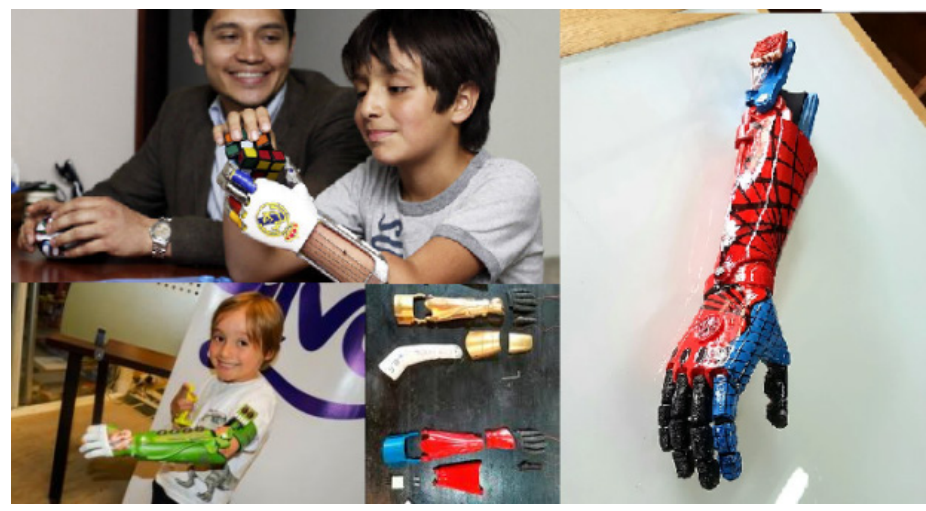

Fuente: elaboración propia.

En esa misma línea, en el 2017, Andrés Cruz Rodríguez, físico de la Universidad Nacional de Colombia, construyó una prótesis que puede ser controlada por medio de ondas cerebrales Agencia de noticias UN, 2017). El cerebro humano funciona mediante impulsos eléctricos, los cuales, a su vez, generan movimientos a lo largo del cuerpo humano. Según el estudio, la región del cerebro en la que Cruz Rodríguez enfocó la investigación fue la frontal para medir las ondas cerebrales que son captadas a través de un electrodo y enviadas a un microprocesador para que este envíe una orden a los actuadores de la prótesis, y así crear movimiento. Para obtener respuesta en la prótesis por medio de las ondas cerebrales, se debe entrar en una etapa de entrenamiento para concentrarse. El tiempo de respuesta del sistema es de $40 \mathrm{~ms}$ y el envío de las señales capturadas por el electrodo se realiza a través de tecnología bluetooth. También se encontró en el estudio que la respuesta de los niños era más efectiva que en adultos. La prótesis fue construida en una impresora 3D y la carga de su batería dura 24 horas para ser nuevamente cargada por 3 horas. Con este sistema se busca llegar a personas en condición de discapacidad con bajos recursos, ya que es más económica que lo encontrado actualmente en el mercado.

Otros adelantos en la fabricación de prótesis se centran en el material utilizado para ellas, como 
es el caso presentado por el programa de diseño industrial de la Universidad ICESI en el 2014 Salazar, 2014). Este desarrollo se centró en utilizar PLA, plástico derivado del almidón de maíz, para la fabricación de una prótesis de miembros inferiores por medio de una impresora 3D. El costo de la prótesis rondaba los 50 dólares, aproximadamente. Además de su bajo costo, también se resalta la capacidad de personalizar la prótesis y el poco tiempo de fabricación.

Alargar vida de las prótesis y evitar una segunda intervención quirúrgica fue la investigación propuesta por la Universidad Industrial de Santander y la empresa Quirúrgicos Especializados (El Tiempo, 2013). La propuesta nace al descubrir que después de 10 años, el usuario de la prótesis comienza a experimentar molestias, dolor, pierde movilidad e incluso se puede someter a una nueva cirugía, por lo cual los investigadores propusieron utilizar biomateriales de calcio para recubrir las prótesis y de esta forma reducir los costos de producción hasta en un $70 \%$,ya que las piezas son importadas.

Continuando con la comodidad del usuario de prótesis, la Universidad de Antioquia planteó una investigación en el 2016 para identificar laceraciones y deformaciones en el muñón (El Tiempo, 2016b), con lo cual se buscó optimizar topografía interna del socket a través de un micromecanizado del polipropileno, para generar rugosidades y texturas que se adapten mejor a la piel.

Debido al flagelo del conflicto armado de las últimas décadas en Colombia, se presentaron 3600 amputados en los últimos 20 años, según estadísticas del Hospital Militar Central, con una inversión de casi 3 millones de dólares en la atención de soldados mutilados (Dussán, 2017). Las mutilaciones en los soldados fueron entre los 18 y 35 años, presentándose 90 \% de las amputaciones en miembros inferiores y el $10 \%$ restante en los miembros superiores.

Figura 8. Víctima del conflicto armado

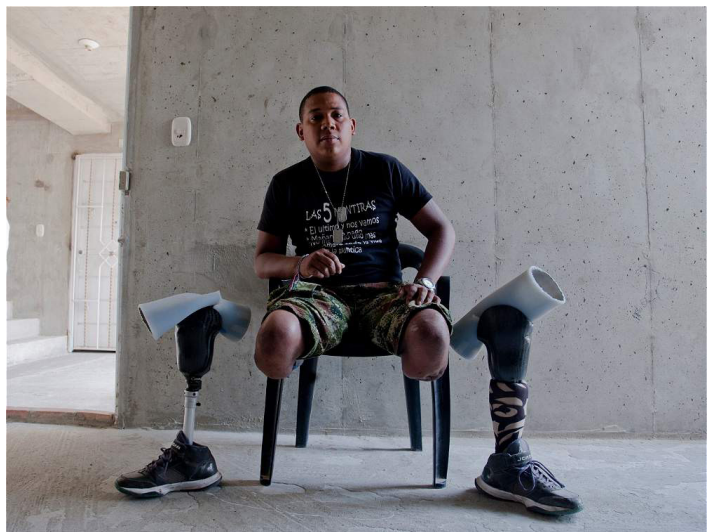

Fuente: Redacción CM\& (2017)

Desde el 2008 se empezaron a desarrollar nuevas tecnologías para afectados por minas antipersonal. En la década de los 80, las prótesis eran en madera y se adquirían en la cruz roja o con entidades alemanas. Más adelante se empezaron a ver prótesis con rodillas mecánicas y de control de movimiento con resortes y elásticos, prótesis modulares, sistemas de suspensión más modernos, con silicona y sistemas de bloqueo. Posteriormente llegaron las prótesis hidráulicas 
o neumáticas, que tenían un $90 \%$ de semejanza en la simulación de movimiento de una extremidad normal. Ya en los últimos años se presentaron las prótesis semi-eléctricas o eléctricas.
Los costos para cada una de estas prótesis fueron los siguientes: 3 millones la de rodilla mecánica, 7 millones la hidráulica y 45 millones la electrónica.

Figura 9. Evolución prótesis

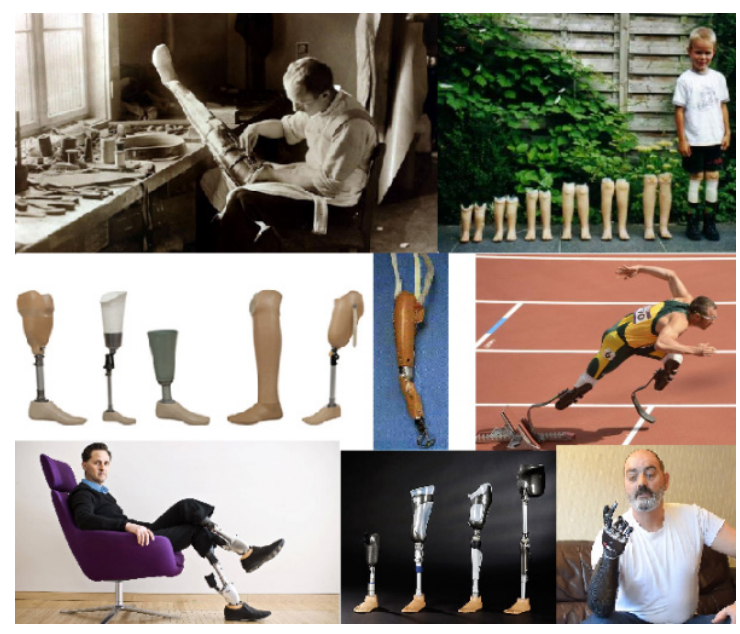

Fuente: elaboración propia.

En Colombia se han hecho adelantos en la fabricación de prótesis gracias a la impresión 3D. Sin embargo, se debe promover investigación en el procesamiento de señales mioléctricas o interfaces cerebro-máquina para controlar las prótesis, lo que produce un mayor confort y destreza en el usuario. Debido al conflicto armado que dejó

\section{Conclusiones}

Uno de los grandes flagelos para Colombia ha sido el conflicto armado. Sin embargo, gracias al acuerdo alcanzado entre el Gobierno Colombiano y las FARC, se ha podido avanzar en la búsqueda de una mejor sociedad. Desde distintos sectores, se ha aportado en el saneamiento de distintas heridas que ha dejado el conflicto, con herramientas como la robótica. Desde las IES, se han desarrollado diferentes sistemas robóticos que detectan y destruyen minas antipersona; se han construido robots para potenciar el agro colombiano, lo que previamente no tenía tanta victimas mutiladas, existe la oportunidad, a través de la investigación, de brindar un mejor futuro con prótesis más avanzadas y más asequibles a los usuarios de estos dispositivos, pues con la importación se incrementa el costo considerablemente.

demasiada relevancia debido a la presencia de grupos armados en áreas rurales; y, finalmente, se han desarrollado prótesis de bajo costo que contribuyen a mejorar la calidad de vida de las personas afectadas por el conflicto armado. Si bien se ha avanzado considerablemente en los distintos frentes presentados en este artículo, aún falta una mayor inversión para realizar un desminado total, potenciar la agroindustria en Colombia y llegar a un mayor número de personas que perdieron algún miembro a causa del conflicto. 


\section{Referencias}

Agencia de noticias UN. (22 de septiembre de 2017). Físico de la UN construye prótesis de mano que puede ser controlada con ondas cerebrales. Caracol Radio. Recuperado de https:/ / caracol.com.co/radio/2017/09/22/tecnologia/1506102949_352748.html

Asociación Colombiana de Fondos de Capital Privado (Colcapital) (junio de 2015). INFORME SECTORIAL - Sector Agroindustrial. Recuperado de https://drive.google.com/ file/d/1mWcTrR9lnw02sNK-zHiqwVv_mvwxVJmJ/view

ASOHOFRUCOL. (2015, Septiembre-Octubre). Drones, los socios de la agricultura de precisión. Frutas \& Hortalizas. Recuperado de https://www.asohofrucol.com.co/img/pdfrevistas/75Lecciones\%20para\%20la\%20productividad.pdf

Bloomberg. (29 de julio de 2017). Colombia quiere convertirse en una potencia agroindustrial. Portafolio. Recuperado de https://www.portafolio.co/economia/ colombia-quiere-convertirse-en-una-potencia-agroindustrial-508227

CNN español (22 dejunio de 2017). El colombiano que fabrica y dona prótesis de superhéroes a niños de bajos recursos. CNN español. Recuperado de https:/ / cnnespanol.cnn.com/2017/06/22/ el-colombiano-que-fabrica-y-dona-protesis-de-superheroes-a-ninos-de-bajos-recursos/

Colombia y las minas antipersonal. (Septiembre de 2000). UNICEF - Colombia. Recuperado de https://www.resdal.org/caeef-resdal/assets/el-salvador---sembrando-minas--cosechando-muerte.pdf

Colorado, J., Mondragon, I., Rodriguez, J., \& Castiblanco, C. (2015). Geo-Mapping and Visual Stitching to Support Landmine Detection Using a Low-Cost UAV. International Journal of Advanced Robotic Systems. DoI: 10.5772/61236

Dussán, Y. (01 de marzo de 2017). Colombian Military Hospital Expands Prosthesis Program. Diálogo. Recuperado de https://dialogo-americas.com/articles/colombian-military-hospital-expands-prosthesis-program/\#.YQhrKI5KiM8

El Colombiano. (22 de julio de 2015). Con robots reinicia desminado humanitario en Antioquia, El Colombiano. Recuperado de https://www.elcolombiano.com/colombia/paz-y-derechos-humanos/con-robots-reinicia-tarea-de-desminado-en-antioquia-XM2358918

El Colombiano (14 de septiembre de 2016). Robot aéreo antiminas fue premiado como el gran invento. El Colombiano. Recuperado de https://www.elcolombiano.com/antioquia/ robot-aereo-antiminas-fue-premiado-como-el-gran-invento-LE4978841 
El Espectador. (12 de julio de 2018). Impacto de la automatización es mayor para países en vías de desarrollo. El Espectador. Recuperado de http://www.elespectador.com/ https:// www.elespectador.com/economia/impacto-de-la-automatizacion-es-mayor-para-paises-en-vias-de-desarrollo-article-799688/

El Mundo. (14 de abril de 2011). 'Arcadio', un robot colombiano que encuentra minas antipersonales. El Mundo. Recuperado de https:/ /www.elmundo.es/america/2011/04/07/colombia/1302207303.html

El Tiempo. (12 de octubre de 2013). Colombia desarrolla prótesis biocompatibles para reducir cirugías. El Tiempo. Recuperado de https://www.eltiempo.com/archivo/documento/ CMS-13118475

El Tiempo. (23 de mayo de 2016a). El 65.8 \% de la tierra apta para sembrar en Colombia no se aprovecha. El Tiempo. Recuperado de https://www.eltiempo.com/archivo/documento/ CMS-16601436

El Tiempo (17 de octubre de 2016b). Lograr prótesis amigables con la piel sí es posible. El Tiempo. Recuperado de https://www.eltiempo.com/archivo/documento/CMS-16728656

Garcia, C. (s.f.). Uso de drones en el cultivo de la caña de azúcar, Colombia.: Tecnicaña. Recuperado de https:/ / tecnicana.org/uso-de-drones-en-el-cultivo-de-la-cana-de-azucar/

Gaviria, F. (2014). El brutal drama de las minas antipersona en Colombia. Las 2 Orillas. Recuperado de https:/ / www.las2orillas.co/el-drama-de-las-minas-antipersona-en-colombia/

González, X. (15 de febrero de 2018). Cultivos de hortalizas serán protegidos por una plataforma robótica. Agronegocios. Recuperado de https://www.agronegocios.co/tecnologia/ cultivos-de-hortalizas-seran-protegidos-por-una-plataforma-robotica-2623240

Gonzoearth (2013). Multispectral GoPro at cloverleaf farm. Recuperado de https://publiclab. org/notes/gonzoearth/07-02-2013/multispectral-gopro-at-cloverleaf-farm

Infodron (2016). Bolivia ya se ayuda de drones en mas del 15\% de su suelo agrícola. Recuperado de https://www.infodron.es/id/2016/08/30/noticia-bolivia-ayuda-drones-suelo-agricola.html

Kickstarter (s.f.). Mine Kafon Drone. Recuperado de https://www.kickstarter.com/projects/ massoudhassani/mine-kafon-drone

Mayorga, D. (28 de noviembre de 2015). Un dron para desminar el país. Pesquisa Javeriana. Recuperado de https://www.javeriana.edu.co/pesquisa/un-dron-para-desminar-el-pais/ 
Medellin, M. J. (2015, 24 de Marzo). La academia, con las botas puestas. El Espectador. Recuperado de https://www.elespectador.com/colombia/mas-regiones/ la-academia-con-las-botas-puestas-article-551287/

Méndez-Fajardo, S.M. y Pérez, B.C. (2015). Posconflicto en Colombia: El rol de la ingeniería. Recuperado de https://docplayer.es/36998084-Posconflicto-en-colombia-el-rol-de-la-ingenieria.html

MinCiencias (03 de junio de 2018). La ruta de la ciencia. Recuperado de https://todoesciencia. minciencias.gov.co/todo_es_ciencia/ruta_ciencia

Redacción CM\& (2017). Carro taller repara las prótesis de los soldados heridos en batalla. Recuperado de https://noticias.canal1.com.co/noticias/ carro-taller-repara-las-protesis-de-los-soldados-heridos-en-batalla/

Salazar, D. (12 de noviembre de 2014). Colombiano desarrolla prótesis económicas en 3D a partir de almidón de maíz. La Información. Recuperado de https:/ / www.lainformacion.com/ economia-negocios-y-finanzas/colombiano-desarrolla-protesis-economicas-en-3d-a-partir-de-almidon-de-maiz_O5wUgsctZMSpjdl7awFHr7/

The Guardian (26 de diciembre de 2015) Drones set to give global farming a makeover. The Guardian. Recuperado de http://www.theguardian.com/ https://www.theguardian.com/ global-development/2015/dec/26/drones-farming-crop-problems-uavs

Victimas de minas antipersonal y municiones sin explosionar. (31 de Mayo de 2018). Organización Descontamina Colombia. Recuperado de http:/ / www.accioncontraminas.gov.co/ Estadisticas/estadisticas-de-victimas\#: :text $=\mathrm{A} \% 20 \mathrm{la} \% 20 \mathrm{fecha} \% 20 \mathrm{de} \% 20$ corte,toda $\% 20$ la \%20historia \%20de\%20Colombia.\&text=En\%20lo\%20corrido\%20del\%20a \%C3\%B1o\%20 $2021 \% 2$ C\%20se \%20han\%20presentado\%2073\%20v\%C3\%ADctimas 